\title{
A Fuzzy Approach Using Generalized Dinkelbach's Algorithm for Multiobjective Linear Fractional Transportation Problem
}

\author{
Nurdan Cetin ${ }^{1}$ and Fatma Tiryaki ${ }^{2}$ \\ ${ }^{1}$ Department of Mathematics, Faculty of Science, Turkish Naval Academy, Tuzla, Istanbul, Turkey \\ ${ }^{2}$ Department of Mathematics, Faculty of Arts and Science, Yildiz Technical University, Davutpasa, Istanbul, Turkey \\ Correspondence should be addressed to Fatma Tiryaki; ftiryaki@yildiz.edu.tr
}

Received 30 September 2013; Revised 14 January 2014; Accepted 21 January 2014; Published 17 March 2014

Academic Editor: Hao-Chun Lu

Copyright (c) 2014 N. Cetin and F. Tiryaki. This is an open access article distributed under the Creative Commons Attribution License, which permits unrestricted use, distribution, and reproduction in any medium, provided the original work is properly cited.

\begin{abstract}
We consider a multiobjective linear fractional transportation problem (MLFTP) with several fractional criteria, such as, the maximization of the transport profitability like profit/cost or profit/time, and its two properties are source and destination. Our aim is to introduce MLFTP which has not been studied in literature before and to provide a fuzzy approach which obtain a compromise Pareto-optimal solution for this problem. To do this, first, we present a theorem which shows that MLFTP is always solvable. And then, reducing MLFTP to the Zimmermann's "min" operator model which is the max-min problem, we construct Generalized Dinkelbach's Algorithm for solving the obtained problem. Furthermore, we provide an illustrative numerical example to explain this fuzzy approach.
\end{abstract}

\section{Introduction}

A lot of research work has been conducted on transportation problems. These problems and their solution techniques play an important role in logistics and supply chain management for reducing cost, improving service quality, and so forth. One of the hot research topics of transportation problems is the use of fuzzy set theory. In 1965, the concept of the fuzzy set was first introduced by Zadeh. Several authors gave the most significant papers on fuzzy sets and their applications. In practical applications, the required data of the real-life problems may be imprecise. Thus, adaptation of fuzzy sets theory in the solution process increases the flexibility and effectiveness of the proposed approaches. There are recent papers by Zadeh published in 2005 and $2008[1,2]$. Fuzzy set theory has become foundation for the development of the fields of transportation systems, especially in their applications. The purpose of this paper is to introduce MLFTP which has not been studied in the literature before and to provide a fuzzy approach for this problem using the Generalized Dinkelbach's Algorithm.

Fractional programming (FP) for single-objective optimization problems was studied extensively from the viewpoint of its application to several real-life problems. For instance, cost benefit analysis in agricultural production planning, faculty and other staff allocation problems for minimizing certain ratios of students' enrolments and stuff structure within academic units of educational institutions, and other optimization problems frequently involve the fractional objectives in a decision situation [3]. As known, a single-objective linear fractional programming is a special case of a nonlinear programming problem and it can be solved by using the variable transformation method [4] or the updated objective function method [5].

Now, since most of the decision making problems in practice are multiobjective in nature, FP with multiplicity of objectives has also been studied by the pioneer researches in the field [3]. Concerning multiobjective linear fractional programming, Kornbluth and Steuer [6, 7], Nykowski and Zołkiewski [8], and Tiryaki [9], and also concerning multiobjective linear fractional programming under fuzzy environment, Luhandjula [10], Sakawa and Yumine [11], Sakawa and Yano [12], and Chakraborty and Gupta [13] proposed different approaches.

In real-life situations, multiple-objective linear transportation problem (MLTP) is more common. A lot of fuzzy 
research work has been conducted on MLTP. In $[14,15]$, Bit et al. developed a procedure using fuzzy programming technique, and an additive fuzzy programming model for the solution of the MLTP, respectively. In [16], Verma et al. used hyperbolic and exponential membership functions to solve the MLTP. In [17], Das et al. proposed a solution procedure of the MLTP where all parameters (the cost coefficients, the source, and destination parameters) have been expressed as interval values by the decision maker. El-Wahed [18] and Li and Lai [19] developed fuzzy approaches to get the compromise solution for MLTP. In [20], Liu and Kao developed a procedure to derive the fuzzy objective value of the fuzzy transportation problem, basing on the extension principle. Ammar and Youness [21] investigated the efficient solutions and stability of MLTP with fuzzy numbers. Abd El-Wahed and Lee [22] presented an interactive fuzzy goal programming approach to determine the preferred compromise solution for MLTP. Fuzzy approaches generally use Zimmermann's fuzzy programming to solve MLTP [17-19]. Due to the easiness of computation, the aggregate operator used in these fuzzy approaches is the "min" operator which does not guarantee a nondominated solution. However, these approaches are more practical than the others-interactive, noninteractive, and goal programming approaches-and have several good features as follows: easy to implement, generally provide the analyst with easy mathematical programming problem, use one of the available software, and so forth. On the other hand, the main disadvantage of fuzzy approaches is that Zimmermann's "min" operator model does not fit the standard form of a transportation problem due to membership function constraints [18].

Transportation problem with fractional objective function is widely used as performance measure in many reallife situations, for example, in the analysis of financial aspects of transportation enterprises and undertaking and in transportation management situations, where an individual or a group of a community is faced with the problem of maintaining good ratios between some crucial parameters concerned with the transportation of commodities from certain sources to various destinations. In transportation problems, examples of fractional objectives include optimization of total actual transportation cost/total standard transportation cost, total return/total investment, risk assets/capital, and total tax/total public expenditure on commodity [23].

To deal with LFTP, it can be observed from literature that few works have been carried out except Bajalinov's work [24]. Bajalinov formulated LFTP in a general form and shortly overviewed its main theoretical results. The author's main computational technique is the Transportation Simplex Method. Besides, he constructed the dual problem of LFTP and finally formulated the main statements of duality theory for this problem. Furthermore, Zenzerovic and Beslic [25] have presented a mathematical model addressing the problem of cargo transport by container ship on the selected route, where certain numbers of containers of various masses and types are chosen out of the total number of containers available in loading port in order to achieve maximum transport profitability, which is an index of operation efficiency of the firm and is expressed as profit/capital, provided maximum payload and transport capacity of container ship.

In this paper, our aim is to obtain a compromise Paretooptimal solution for MLFTP by means of Zimmermann's "min" operator. Using Generalized Dinkelbach's Algorithm, MLFTP that has nonlinear structure is reduced to a sequence of linear programming problems. However, the solution generated by "min" operator does not guarantee Paretooptimality. For this reason, we offer a Pareto-optimality test to obtain a compromise Pareto-optimal solution.

This paper is organized as follows. Section 2 presents MLFTP formulation and introduces the definitions of Paretooptimal, weakly Pareto-optimal, and compromise solution for MLFTP. Section 3 explains our fuzzy approach to the MLFTP using Generalized Dinkelbach's Algorithm and then how the Pareto-optimality test is conducted. Section 4 gives an illustrative example. The paper ends with some concluding remarks in Section 5.

\section{Multiobjective Linear Fractional Transportation Problem}

Assume that there are $m$ sources and $n$ destinations. At each source, let $a_{i}(i=1,2, \ldots, m)$ be the amount of homogenous products which are transported to $n$ destinations to satisfy the demand for $b_{j}(j=1,2, \ldots, n)$ units of the product there. Let variable $x_{i j}$ be units of goods shipped from source $i$ to destination $j$. For the objective function $z_{q}(\mathbf{x}),(q=$ $1, \ldots, Q)$, profit matrix $p_{q}=\left[p_{i j}^{q}\right]_{m \times n}$ which determines the profit $p_{i j}^{q}$ gained from shipment from $i$ to $j$; cost matrix $d_{q}=$ $\left[d_{i j}^{q}\right]_{m \times n}$ which determines the cost $d_{i j}^{q}$ per unit of shipment from $i$ to $j$; scalars $p_{0}^{q}, d_{0}^{q}$, which determine some constant profit and cost, respectively. Then the mathematical model of the MLFTP can be formulated as follows:

$$
\begin{aligned}
& \max \quad z_{q}(\mathbf{x})=\frac{p_{q}(\mathbf{x})}{d_{q}(\mathbf{x})}=\frac{\sum_{i=1}^{m} \sum_{j=1}^{n} p_{i j}^{q} x_{i j}+p_{0}^{q}}{\sum_{i=1}^{m} \sum_{j=1}^{n} d_{i j}^{q} x_{i j}+d_{0}^{q},} \\
& q=1, \ldots, Q, \\
& \text { s.t. } \quad \sum_{j=1}^{n} x_{i j} \leq a_{i}, \quad i=1,2, \ldots, m, \\
& \quad \sum_{i=1}^{m} x_{i j} \geq b_{j}, \quad j=1,2, \ldots, n, \\
& \quad x_{i j} \geq 0, \quad i=1,2, \ldots, m ; j=1,2, \ldots, n,
\end{aligned}
$$

where $\mathbf{z}_{q}(\mathbf{x})=\left(z_{1}(\mathbf{x}), z_{2}(\mathbf{x}), \ldots, z_{\mathrm{Q}}(\mathbf{x})\right)$ is a vector of objective functions (criteria vector) and "max" means that all efficient solutions are to be found. We suppose that $d_{q}(\mathbf{x})>0, q=$ $1, \ldots, Q$ and $\forall \mathbf{x}=\left(x_{i j}\right) \in S$, where $S \neq \emptyset$ denotes a convex and compact feasible set defined by constraints (1b)-(1d); the functions $p_{q}(\mathbf{x})$ and $d_{q}(\mathbf{x})$ are continuous on $S$. Further, we 
assume that $a_{i}>0, \forall i ; b_{j}>0, \forall j ; p_{i j}^{q}>0, d_{i j}^{q}>0, p_{0}^{q}>0$, $d_{0}^{q}>0$ for all $i, j$, and

$$
\sum_{i=1}^{m} a_{i}
$$

$\geq \sum_{j=1}^{n} b_{j} \quad$ (total supply is not less than total demand).

The inequality (2) is treated as a necessary and sufficient condition for the existence of a feasible solution to problems (1a)-(1d).

Theorem 1. MLFTP is solvable if and only if the above inequality (2) holds.

Proof.

Necessity. Suppose that MLFTP is solvable and $\mathbf{x}$ is its basic feasible solution. Adding together separately supply constraints (1b) for all indices $i=1,2, \ldots, m$, demand constraints (1c) for all indices $j=1,2, \ldots, n$, we obtain

$$
\sum_{i=1}^{m} \sum_{j=1}^{n} x_{i j} \leq \sum_{i=1}^{m} a_{i}, \quad \sum_{j=1}^{n} \sum_{i=1}^{m} x_{i j} \geq \sum_{j=1}^{n} b_{j}
$$

respectively. Since the left-hand sides of the inequalities obtained are exactly the same, total demand is smaller or equal to total supply. Hence, inequality (2) holds.

Sufficiency. Suppose now that inequality (2) holds. We have to show that in this case, feasible set $S$ of problems (1a)-(1d) is not empty and all objective functions $z_{q}(\mathbf{x})$ and $q=1, \ldots, Q$ over set $S$ are bounded. Let

$$
x_{i j}^{\prime}=\frac{a_{i} b_{j}}{R}, \quad i=1,2, \ldots, m ; j=1,2, \ldots, n,
$$

where

$$
R=\sum_{j=1}^{n} b_{j}>0
$$

We will show that $x_{i j}^{\prime}$ is a feasible solution of problems (1a)-(1d). Indeed, we have

$$
\begin{gathered}
\sum_{j=1}^{n} x_{i j}^{\prime}=\sum_{j=1}^{n} \frac{a_{i} b_{j}}{R}=\frac{a_{i}}{R} \sum_{j=1}^{n} b_{j}=\frac{a_{i}}{R} R=a_{i}, \quad i=1,2, \ldots, m, \\
\sum_{i=1}^{m} x_{i j}^{\prime}=\sum_{i=1}^{m} \frac{b_{j} a_{i}}{R}=\frac{b_{j}}{R} \sum_{i=1}^{m} a_{i} \\
\begin{array}{c}
\text { (inequality 2) } \\
\geq
\end{array} \frac{b_{j}}{R} \sum_{j=1}^{n} b_{j}=\frac{b_{j}}{R} R=b_{j}, \\
j=1,2, \ldots, n,
\end{gathered}
$$

respectively. Hence, constraints (1b) and (1c) are satisfied by $x_{i j}^{\prime}$. Further, since $a_{i}>0, \forall i$ and $b_{j}>0, \forall j$, it ensures us that $x_{i j}^{\prime} \geq 0$ for all indices $i, j$. Hence, we have shown that feasible set $S$ is not empty and contains at least one feasible solution $\mathbf{x}^{\prime}=\left(x_{i j}^{\prime}\right)$.

From (1b) and (1d) we have that

$$
0 \leq x_{i j} \leq a_{i}, \quad i=1,2, \ldots, m ; j=1,2, \ldots, n .
$$

The latter means that feasible set is bounded.

Finally, since $p_{q}(\mathbf{x})$ and $d_{q}(\mathbf{x}), q=1, \ldots, Q$, are linear functions and $d_{q}(\mathbf{x})>0$ and $q=1, \ldots, Q$ are bounded over feasible set $S$, it means that all objective function $z_{q}(\mathbf{x})$ and $q=1, \ldots, Q$ are also bounded over set $S$.

Hence, the MLFTP is solvable.

Definition 2. If total demand equals total supply, that is,

$$
\sum_{i=1}^{m} a_{i}=\sum_{j=1}^{n} b_{j}, \quad \text { (balanced condition) }
$$

then the MLFTP is said to be a balanced transportation problem.

If a transportation problem has a total supply that is strictly less than total demand, the problem has no feasible solution. In this situation, it is sometimes desirable to allow the possibility of leaving some demand unmet. In such a case, we can balance a transportation problem by creating a dummy supply point that has a supply equal to the amount of unmet demand and associating a penalty with it.

By converting inequalities (lb) and (1c) into equalities, MLFTP in a canonical form can be defined.

Theorem 3. MLFTP in a canonical form is solvable if and only if the above balanced condition (8) holds.

Proof. This theorem can be proved by a procedure similar to Theorem 1 .

In the context of multiple criteria, definitions of efficient and nondominated or Pareto-optimal solutions are used instead of the optimal solution concept in single-objective programming. In the multiple-objective linear fractional programming, the concept of the strongly efficient solution which corresponds to the standard definition of the efficient solution in multiobjective linear programming is insufficient, and therefore the weakly efficient concept is considered. In theory, finding the strongly efficient solutions is desired. However, in practice, solution algorithms tend to find weakly efficient solutions. This is because, vertexes of the weakly Pareto-optimal solution set $\left(E^{W}\right)$ construct a connected graph [6-9]. Now we present the following definitions of Pareto-optimal, weakly Pareto-optimal, and compromise solution for MLFTP.

Definition 4 (Pareto-Optimal Solution for MLFTP). The point $\overline{\mathbf{x}} \in S$ is a Pareto-optimal (strongly efficient or nondominated) solution if and only if there does not exist another $\mathbf{x} \in S$ such that $z_{q}(\mathbf{x}) \geq z_{q}(\overline{\mathbf{x}})$ for all $q=1, \ldots, Q$ and $z_{q}(\mathbf{x})>z_{q}(\overline{\mathbf{x}})$ for at least one $q$. 
Definition 5 (Weakly Pareto-Optimal Solution for MLFTP). The point $\overline{\mathbf{x}} \in S$ is a weakly Pareto-optimal solution if and only if there does not exist another $\mathbf{x} \in S$ such that $z_{q}(\mathbf{x})>z_{q}(\overline{\mathbf{x}})$ for all $q=1, \ldots, Q$.

According to these definitions, $E^{S} \subset E^{W}$, where $E^{S}$ denotes the set of Pareto-optimal solutions.

Definition 6 (Compromise Solution [22, 26, 27] for MLFTP). A feasible point $\overline{\mathbf{x}} \in S$ is a compromise solution if and only if $\overline{\mathbf{x}} \in E^{W}$ and $\mathbf{Z}(\overline{\mathbf{x}}) \geq \wedge_{\mathbf{x} \in S} \mathbf{Z}(\mathbf{x})$ where $\mathbf{Z}(\mathbf{x})=\left(z_{1}(\mathbf{x})\right.$, $\left.z_{2}(\mathbf{x}), \ldots, z_{\mathrm{Q}}(\mathbf{x})\right)$ and $\wedge$ stands for "min" operator.

According to Definition 6, (i) a solution should be weakly efficient to be a compromise solution; (ii) the feasible solution vector $\overline{\mathbf{x}}$ should have the minimum deviation from the ideal point than any other point in $S$. The compromise solution is the closest solution to the ideal one that maximizes the underlying utility function of the decision maker. So this compromise solution is obtained from the max-min problem.

In general, an optimal solution which simultaneously maximizes all the objective functions in MLFTP does not always exist when the objective functions conflict with each other. When a certain Pareto-optimal solution is selected, any improvement of one objective function can be achieved only at the expense of at least one of the other objective functions. Thus, the above Definition 4 for MLFTP is the same as the definition of the Pareto-optimality in multiobjective linear programming.

\section{Solution Procedure}

In this section, using Zimmermann's “min” operator, we will give a fuzzy approach to obtain a compromise Pareto-optimal solution for MLFTP.

3.1. Constructing the Membership Functions of the Objectives. There are several membership functions in the literature, for example, linear, hyperbolic, and piecewise-linear, and so forth [28]. For simplicity, in this paper, we adopt a linear membership function. We can define the membership function $\mu_{q}\left(z_{q}\right)$ corresponding to $q$ th objective as

$$
\mu_{q}\left(z_{q}\right)= \begin{cases}0, & z_{q}<z_{q}^{m}, \\ \frac{z_{q}-z_{q}^{m}}{z_{q}^{*}-z_{q}^{m}}, & z_{q}^{m} \leq z_{q} \leq z_{q}^{*}, \\ 1, & z_{q}^{*}<z_{q},\end{cases}
$$

where $\max _{\mathbf{x} \in S} z_{q}(\mathbf{x})=z_{q}^{*}$ and $\min _{\mathbf{x} \in S} z_{q}(\mathbf{x})=z_{q}^{m}, q=1, \ldots, Q$, denote the values of the objective function $z_{q}(\mathbf{x})$ such that the degrees of membership function are 1 and 0 , respectively. The membership function is linear and strictly monotone increasing with respect to $z_{q}$ in the interval $\left[z_{q}^{m}, z_{q}^{*}\right]$. To construct (9) we note that we solved $2 Q$ times a single-objective linear fractional transportation programming problem.
First of all, by using the "min" fuzzy operator model proposed by Zimmermann [29], the following problem is solved for MLFTP:

$$
\begin{aligned}
& \max _{\mathbf{x} \in S} \min _{1 \leq q \leq Q} \mu_{q}\left(z_{q}(\mathbf{x})\right) \\
& \text { s.t. } \quad \mathbf{x} \in S .
\end{aligned}
$$

By introducing the auxiliary variable $\lambda$,

$$
\min \mu_{q}\left(z_{q}\right)=\lambda \Longrightarrow \mu_{q}\left(z_{q}\right) \geq \lambda,
$$

this problem can be transformed into the following equivalent maximization problem:

$$
\begin{array}{ll}
\max & \lambda \\
\text { s.t. } & \mu_{q}\left(z_{q}\right) \geq \lambda, \\
& \mathbf{x} \in S .
\end{array}
$$

Here, since the membership function $\mu_{q}\left(z_{q}\right)$ is the strictly monotone increasing for objective $z_{q}$ in the closed interval $\left[z_{q}^{m}, z_{q}^{*}\right]$, from (12) we have

$$
\begin{array}{ll}
\max & \lambda \\
\text { s.t. } & z_{q} \geq \mu_{q}^{-1}(\lambda), \\
& \mathbf{x} \in S,
\end{array}
$$

where

$$
\mu_{q}^{-1}(\lambda)=\inf \left\{z_{q} \mid \mu_{q}\left(z_{q}\right) \geq \lambda\right\} .
$$

Problem (13) or (10) is the "min" operator model for MLFTP and also a nonlinear programming model. Its optimal objective value $\lambda^{*}$ denotes the maximum of the least satisfaction levels among all objectives expressed as profit/cost or profit/time and so forth, simultaneously, and it can also be interpreted as the "most basic satisfaction" that each objective in this transportation system can attain.

The transportation problem in (10) is in nature of a generalized linear fractional programming problem. Therefore, our fuzzy approach for solving this transportation problem use an iterative algorithm based on Generalized Dinkelbach's Algorithm [24, 30, 31]. It is known that "min-" operator model is a noncompensatory model [27]. So, problem (10) produces the weakly Pareto-optimal solution for MLFTP, but it does not guarantee to get a Pareto-optimal solution. For this reason, the Pareto-optimality test given in Section 3.3 is applied to obtain the Pareto-optimal solution.

3.2. Generalization of Dinkelbach's Algorithm for MLFTP. One of the most popular and general strategies for fractional programming (not necessary linear) is the parametric approach described by W. Dinkelbach. In the case of linear fractional programming, this algorithm reduces the solution of a sequence of linear programming problems [24]. Therefore, it is efficient and robust in practice, and so it is preferable 
for MLFTP. Furthermore, even if the Dinkelbach's algorithm may require longer solution time than metaheuristic, it allows getting the optimal solutions for several problems and verifying the efficiency of the metaheuristic methods [32].

In this section, we consider the max-min problem (10). For simplicity, let

$$
\mu_{q}\left(z_{q}(\mathbf{x})\right)=\frac{z_{q}-z_{q}^{m}}{z_{q}^{*}-z_{q}^{m}}=\frac{P_{q}(\mathbf{x})}{D_{q}(\mathbf{x})}, \quad q=1, \ldots, Q
$$

be in the interval $\left[z_{q}^{m}, z_{q}^{*}\right]$ and then problem (10) is equivalent to

$$
\bar{\lambda}=\max _{\mathbf{x} \in S} \min _{1 \leq q \leq Q}\left\{\frac{P_{q}(\mathbf{x})}{D_{q}(\mathbf{x})}\right\} .
$$

We call this problem a generalized linear fractional transportation problem. Since all membership functions are linear fractional functions, this problem is solved by Generalized Dinkelbach's Algorithm. This algorithm corresponds to solving a sequence of the following parametric problems:

$$
F(\lambda)=\max _{\mathbf{x} \in S} \min _{1 \leq q \leq Q}\left(P_{q}(\mathbf{x})-\lambda D_{q}(\mathbf{x})\right)
$$

Before discussing the method, the following two lemmas will be introduced. These statements establish relations between the problem (16) and the problem (17) with parametric function $F(\lambda)$.

Lemma 7 (see [24]). Let

$$
\bar{\lambda}=\max _{\mathbf{x} \in S} \min _{1 \leq q \leq Q}\left\{\frac{P_{q}(\mathbf{x})}{D_{q}(\mathbf{x})}\right\},
$$

then

(1) parametric function $F(\lambda)>-\infty$; moreover, $F(\lambda)$ is lower semicontinuous and nondecreasing;

(2) $F(\lambda)>0$ if and only if $\lambda<\bar{\lambda}$;

(3) $F(\bar{\lambda}) \leq 0$;

(4) if problem (16) is solvable then $F(\bar{\lambda})=0$;

(5) if $F(\bar{\lambda})=0$ then problem (16) and problem (17) have the same set of optimal solutions (which may be empty).

Theorem 8 (see [33]). Assume that $S$ is compact. The following results hold.

(a) Equations (16) and (17) always have an optimal solution, $\bar{\lambda}$ is finite, and $F(\bar{\lambda})=0$. Hence, $F(\lambda)=0$ implies $\lambda=\bar{\lambda}$.

(b) Parametric function $F$ is finite, continuous, and increasing on $\mathbb{R}$. (c) The sequence $\left\{\lambda^{r}\right\}$, if not finite, converges linearly to $\bar{\lambda}$, and each convergent subsequence of $\left\{\mathbf{x}^{r}\right\}$ converges to an optimal solution of (16).

This lemma and theorem provide the necessary theoretical basis for a generalization of Dinkelbach's algorithm. Algorithm is suggested that finds the constrained maximum of the minimum of finitely many ratios. The method involves a sequence of linear subproblems if the ratios are linear.

Now we can give Generalized Dinkelbach's Algorithm for MLFTP.

Algorithm 9 (Generalized Dinkelbach's Algorithm for MLFTP).

Step 0 . Set $r:=0$.

Step 1. Take an arbitrary feasible solution $\mathbf{x}^{r}\left(=\mathbf{x}^{0}\right) \in S$, and

$$
\text { compute } \lambda^{(0)}=\min _{1 \leq q \leq Q}\left\{\frac{P_{q}\left(\mathbf{x}^{(0)}\right)}{D_{q}\left(\mathbf{x}^{(0)}\right)}\right\} \text {. }
$$

Step 2. Solve the following problem:

$$
\begin{array}{ll}
\max & t \\
\text { s.t. } & \frac{1}{D_{q}\left(\mathbf{x}^{(r)}\right)}\left[P_{q}(\mathbf{x})-\lambda^{r} D_{q}(\mathbf{x})\right] \geq t, \quad q=1, \ldots, Q, \\
& \mathbf{x} \in S .
\end{array}
$$

Let the obtained solution be $\mathbf{x}^{r+1}$.

Step 3. If $t<\delta$ (where $\delta>0$ is the termination parameter) then the current solution $\mathbf{x}^{*}=\mathbf{x}^{r+1}$ is an optimal solution of the problem (16) and $\lambda^{(r)}$ is its optimal value, Stop. Otherwise, $\lambda^{(r+1)}:=\min _{1 \leq q \leq Q}\left\{P_{q}\left(\mathbf{x}^{\mathbf{r}+1}\right) / D_{q}\left(\mathbf{x}^{\mathbf{r}+1}\right)\right\}$ is computed. Set $r:=$ $r+1$ and return to Step 2 .

The convergence of sequence $\left\{\lambda^{r}\right\}$ generated from parametric problem $F\left(\lambda^{r}\right)$ in Step 2 is guaranteed by the following properties of the sequence:

(i) for all $r \geq 0, \lambda^{(r)}=\min _{1 \leq q \leq Q} \mu_{q}\left(z_{q}\left(\mathbf{x}^{r}\right)\right) \leq \lambda^{*}$;

(ii) the sequence $\left\{\lambda^{r}\right\}$ is monotone increasing,

where $\lambda^{*}=\max _{\mathbf{x} \in S} \min _{1 \leq q \leq \mathrm{Q}} \mu_{q}\left(z_{q}(\mathbf{x})\right)$.

3.3. Pareto-Optimality Test. A current solution $\mathbf{x}^{*}$ obtained from Step 3 of the problem (10) is weakly Pareto-optimal compromise solution for problems (1a)-(1d). If an alternative optimal solution which has the same optimal value $\lambda$ does not exist, then $\mathbf{x}^{*}$ is also Pareto-optimal as well. On the other hand, if there is an alternative optimal solution, we 
conduct the following Pareto-optimality test in order to find the Pareto-optimal solution $[6,34]$ :

$$
\begin{array}{ll}
\max & \sum_{q=1}^{Q} \varepsilon_{q} \\
\text { s.t. } & z_{q}(\mathbf{x})-\varepsilon_{q} \geq z_{q}\left(\mathbf{x}^{*}\right), \quad q=1, \ldots, Q, \\
& \mathbf{x} \in S, \\
& \mathbf{x} \geq \mathbf{0}, \quad \varepsilon_{q} \geq 0, q=1, \ldots, Q .
\end{array}
$$

By doing the necessary substitution, we solve the following problem equivalently:

$$
\begin{array}{ll}
\max & \sum_{q=1}^{Q} \widehat{\varepsilon}_{q} \\
\text { s.t. } \quad\left(\sum_{i=1}^{m} \sum_{j=1}^{n} p_{i j}^{q}-\sum_{i=1}^{m} \sum_{j=1}^{n} d_{i j}^{q} z_{q}^{*}\right) \cdot \mathbf{x}-\widehat{\varepsilon}_{q} \\
\geq d_{0}^{q} \cdot z_{q}^{*}-p_{0}^{q}, \quad q=1, \ldots, Q, \\
\mathbf{x} \in S, \quad \\
\mathbf{x} \geq \mathbf{0}, \quad \widehat{\varepsilon}_{q} \geq 0, \quad q=1, \ldots, Q .
\end{array}
$$

If $\max \sum_{q=1}^{\mathrm{Q}} \widehat{\varepsilon}_{q}=0, \mathbf{x}^{*}$ is the Pareto-optimal solution. Provided that $\max \sum_{q=1}^{\mathrm{Q}} \widehat{\varepsilon}_{q}>0$, the solution to the last linear programming problem is $\overline{\mathbf{x}}$, we resolve this Pareto linear programming problem by replacing $\mathbf{x}^{*}$ with $\overline{\mathbf{x}}$.

\section{Numerical Example}

Let us apply the fuzzy approach presented above to the following numerical example. Consider

$$
\begin{aligned}
& \max z_{1}(\mathbf{x})=\frac{x_{11}+2 x_{12}+8 x_{21}+6 x_{22}+4}{x_{11}+3 x_{12}+x_{21}+2 x_{22}+2} \\
& \max z_{2}(\mathbf{x})=\frac{2 x_{11}+4 x_{12}+10 x_{21}+8 x_{22}+6}{x_{11}+2 x_{12}+3 x_{21}+x_{22}+4} \\
& \max z_{3}(\mathbf{x})=\frac{6 x_{11}+x_{12}+4 x_{21}+5 x_{22}+8}{2 x_{11}+x_{12}+x_{21}+3 x_{22}+5}
\end{aligned}
$$

s.t.

Supply constraints :

$$
\begin{aligned}
& x_{11}+x_{12} \leq 150 \\
& x_{21}+x_{22} \leq 250
\end{aligned}
$$

Demand constraints :

$$
\begin{aligned}
& x_{11}+x_{21} \geq 50 \\
& x_{12}+x_{22} \geq 350 \\
& \mathbf{x}=\left(x_{11}, x_{12}, x_{21}, x_{22}\right) \geq \mathbf{0} .
\end{aligned}
$$

TABle 1: The individual maxima and minima and corresponding solutions.

\begin{tabular}{lccc}
\hline & $z_{1}$ & $z_{2}$ & $z_{3}$ \\
\hline$z^{*}$ & 2.111 & 4.972 & 1.736 \\
$z^{m}$ & 2.059 & 4.138 & 1.687 \\
$x_{1}^{*}(0,150,50,200)$ & 2.111 & 4.138 & 1.687 \\
$x_{1}^{m}(50,100,0,250)$ & 2.059 & 4.972 & 1.736 \\
$x_{2}^{*}(50,100,0,250)$ & 2.059 & 4.972 & 1.736 \\
$x_{2}^{m}(0,150,50,200)$ & 2.111 & 4.138 & 1.687 \\
$x_{3}^{*}(50,100,0,250)$ & 2.059 & 4.972 & 1.736 \\
$x_{3}^{m}(0,150,50,200)$ & 2.111 & 4.138 & 1.687
\end{tabular}

Three linear fractional transportation problems are solved as nonlinear programming problems directly by using any available nonlinear programming package, for example, GAMS [35] and Gino or super Gino, or they are reduced to the linear programming problems with Charnes and Cooper variable transformation [4] and then solved by using the LP solver package such as winqsb and LINDO. The individual maxima and minima and corresponding solutions for three objectives are shown in Table 1. For example, the individual results for the objective function $z_{1}$ are $z_{1}^{*}=2.111$ at $x_{1}^{*}(0,150,50,200)$ and $z_{1}^{m}=2.059$ at $x_{1}^{m}(50,100,0,250)$.

By using (9) and Table 1, the membership functions of 3 objective functions in the interval $\left[z_{q}^{m}, z_{q}^{*}\right]$ can be designed as follows:

$$
\begin{aligned}
\mu_{1}\left(z_{1}(\mathbf{x})\right) & \\
= & \left(-20.366 x_{11}-80.328 x_{12}+114.251 x_{21}\right. \\
& \left.+36.193 x_{22}-2.269\right) \\
& \times\left(x_{11}+3 x_{12}+x_{21}+2 x_{22}+2\right)^{-1}, \\
\mu_{2}\left(z_{2}(\mathbf{x})\right) & \\
= & -2.563 x_{11}-5.127 x_{12}-2.894 x_{21} \\
& \left.+4.631 x_{22}-12.652\right) \\
& \times\left(x_{11}+2 x_{12}+3 x_{21}+x_{22}+4\right)^{-1}, \\
\mu_{3}\left(z_{3}(\mathbf{x})\right) & \left(53.591 x_{11}-14.02 x_{12}+47.204 x_{21}\right. \\
& \left.\quad-1.245 x_{22}-8.877\right) \\
& \times\left(2 x_{11}+x_{12}+x_{21}+3 x_{22}+5\right)^{-1} .
\end{aligned}
$$

"min" operator model corresponding to (10) for (23) will be in the form as follows:

$\max \lambda$

$$
\begin{aligned}
& \mu_{1}\left(z_{1}(\mathbf{x})\right) \geq \lambda \\
& \Longrightarrow\left(-20.366 x_{11}-80.328 x_{12}+114.251 x_{21}\right.
\end{aligned}
$$




$$
\begin{gathered}
\left.+36.193 x_{22}-2.269\right) \\
\times\left(x_{11}+3 x_{12}+x_{21}+2 x_{22}+2\right)^{-1} \geq \lambda, \\
\mu_{2}\left(z_{2}(\mathbf{x})\right) \geq \lambda \\
\Longrightarrow\left(-2.563 x_{11}-5.127 x_{12}-2.894 x_{21}\right. \\
\left.+4.631 x_{22}-12.652\right) \\
\quad \times\left(x_{11}+2 x_{12}+3 x_{21}+x_{22}+4\right)^{-1} \geq \lambda, \\
\mu_{3}\left(z_{3}(\mathbf{x})\right) \geq \lambda \\
\Longrightarrow\left(53.591 x_{11}-14.02 x_{12}+47.204 x_{21}\right. \\
\left.-1.245 x_{22}-8.877\right) \\
\times\left(2 x_{11}+x_{12}+x_{21}+3 x_{22}+5\right)^{-1} \geq \lambda,
\end{gathered}
$$

Supply constraints:

$$
\begin{aligned}
& x_{11}+x_{12} \leq 150, \\
& x_{21}+x_{22} \leq 250
\end{aligned}
$$

Demand constraints:

$$
\begin{aligned}
& x_{11}+x_{21} \geq 50, \\
& x_{12}+x_{22} \geq 350, \\
& \mathbf{x}=\left(x_{11}, x_{12}, x_{21}, x_{22}\right) \geq \mathbf{0} .
\end{aligned}
$$

For solving problem (25) the Generalized Dinkelbach's Algorithm proceeds as follows:

Step 0. Set $r:=0$.

Step 1. Let a feasible solution $\mathbf{x}^{(0)}=(0,150,50,200) \in S$ to $\max \left\{\mathbf{0}^{T} \mathbf{x} \mid \mathbf{x} \in S\right\}$. The satisfactory levels of the objectives corresponding to $\mathbf{x}^{(0)}$ are $\mu_{1}\left(z_{1}\left(\mathbf{x}^{(0)}\right)\right)=P_{1}\left(\mathbf{x}^{(0)}\right) / D_{1}\left(\mathbf{x}^{(0)}\right)=1$, $\mu_{2}\left(z_{2}\left(\mathbf{x}^{(0)}\right)\right)=P_{2}\left(\mathbf{x}^{(0)}\right) / D_{2}\left(\mathbf{x}^{(0)}\right)=0$, and $\mu_{3}\left(z_{3}\left(\mathbf{x}^{(0)}\right)\right)=$ $P_{3}\left(\mathbf{x}^{(0)}\right) / D_{3}\left(\mathbf{x}^{(0)}\right)=0$, and we compute the minimal satisfactory level of them as

$$
\lambda^{(0)}=\min \left\{\frac{P_{1}\left(\mathbf{x}^{(0)}\right)}{D_{1}\left(\mathbf{x}^{(0)}\right)}, \frac{P_{2}\left(\mathbf{x}^{(0)}\right)}{D_{2}\left(\mathbf{x}^{(0)}\right)}, \frac{P_{3}\left(\mathbf{x}^{(0)}\right)}{D_{3}\left(\mathbf{x}^{(0)}\right)}\right\}=0 .
$$

Step 2. Now, for $\lambda^{(0)}=0$, we construct the following problem: $\max t$

$$
\begin{aligned}
& \frac{1}{D_{1}\left(\mathbf{x}^{(0)}\right)}\left[P_{1}(\mathbf{x})-\lambda^{(0)} D_{1}(\mathbf{x})\right] \geq t, \\
& \frac{1}{D_{2}\left(\mathbf{x}^{(0)}\right)}\left[P_{2}(\mathbf{x})-\lambda^{(0)} D_{2}(\mathbf{x})\right] \geq t,
\end{aligned}
$$

$$
\begin{aligned}
& \frac{1}{D_{3}\left(\mathbf{x}^{(0)}\right)}\left[P_{3}(\mathbf{x})-\lambda^{(0)} D_{3}(\mathbf{x})\right] \geq t, \\
& x_{11}+x_{12} \leq 150, \quad x_{21}+x_{22} \leq 250, \\
& x_{11}+x_{21} \geq 50, \quad x_{12}+x_{22} \geq 350 \\
& \mathbf{x}=\left(x_{11}, x_{12}, x_{21}, x_{22}\right) \geq \mathbf{0} .
\end{aligned}
$$

that is,

$$
\begin{gathered}
\max t \\
0.001\left(-20.366 x_{11}-80.328 x_{12}+114.251 x_{21}\right. \\
\left.+36.193 x_{22}-2.269\right) \geq t \\
0.002\left(-2.563 x_{11}-5.127 x_{12}-2.894 x_{21}\right. \\
\left.+4.631 x_{22}-12.652\right) \geq t \\
0.001\left(53.591 x_{11}-14.02 x_{12}+47.204 x_{21}\right. \\
\left.-1.245 x_{22}-8.877\right) \geq t \\
x_{11}+x_{12} \leq 150, \quad x_{21}+x_{22} \leq 250 \\
x_{11}+x_{21} \geq 50, \quad x_{12}+x_{22} \geq 350 \\
\mathbf{x}=\left(x_{11}, x_{12}, x_{21}, x_{22}\right) \geq \mathbf{0} .
\end{gathered}
$$

Solving this problem, we obtain $\mathbf{x}^{(1)}=\left(x_{11}, x_{12}, x_{21}, x_{22}\right)=$ $(24.165,125.835,25.835,224.165)$.

Step 3. Let $\delta$, the termination parameter, take the value of $10^{-3}$. Since $t=0.462>\delta$ the satisfactory levels for $\mathbf{x}^{(1)}$ are $\mu_{1}\left(z_{1}\left(\mathbf{x}^{(1)}\right)\right)=P_{1}\left(\mathbf{x}^{(1)}\right) / D_{1}\left(\mathbf{x}^{(1)}\right)=0.527$, $\mu_{2}\left(z_{2}\left(\mathbf{x}^{(1)}\right)\right)=P_{2}\left(\mathbf{x}^{(1)}\right) / D_{2}\left(\mathbf{x}^{(1)}\right)=0.419$, and $\mu_{3}\left(z_{3}\left(\mathbf{x}^{(1)}\right)\right)=$ $P_{3}\left(\mathbf{x}^{(1)}\right) / D_{3}\left(\mathbf{x}^{(1)}\right)=0.527$, and their minimal is $\lambda^{(1)}=$ $\min \left\{P_{1}\left(\mathbf{x}^{(1)}\right) / D_{1}\left(\mathbf{x}^{(1)}\right), P_{2}\left(\mathbf{x}^{(1)}\right) / D_{2}\left(\mathbf{x}^{(1)}\right), P_{3}\left(\mathbf{x}^{(1)}\right) / D_{3}\left(\mathbf{x}^{(1)}\right)\right\}=$ 0.419 . Let $r:=r+1$.

Step 2. For $\lambda^{(1)}=0.419$, we construct problem

$\max t$

$$
\begin{aligned}
& \frac{1}{D_{1}\left(\mathbf{x}^{(1)}\right)}\left[P_{1}(\mathbf{x})-\lambda^{(1)} D_{1}(\mathbf{x})\right] \geq t, \\
& \frac{1}{D_{2}\left(\mathbf{x}^{(1)}\right)}\left[P_{2}(\mathbf{x})-\lambda^{(1)} D_{2}(\mathbf{x})\right] \geq t, \\
& \frac{1}{D_{3}\left(\mathbf{x}^{(1)}\right)}\left[P_{3}(\mathbf{x})-\lambda^{(1)} D_{3}(\mathbf{x})\right] \geq t, \\
& x_{11}+x_{12} \leq 150, \quad x_{21}+x_{22} \leq 250, \\
& x_{11}+x_{21} \geq 50, \quad x_{12}+x_{22} \geq 350, \\
& \mathbf{x}=\left(x_{11}, x_{12}, x_{21}, x_{22}\right) \geq \mathbf{0},
\end{aligned}
$$


TABLE 2: The results corresponding to five iterations for the problem (25).

\begin{tabular}{lccccc}
\hline Iteration $r$ & $x^{(r)}$ & $\mu_{1}\left(x^{(r)}\right)$ & $\mu_{2}\left(x^{(r)}\right)$ & $\mu_{3}\left(x^{(r)}\right)$ & $\lambda^{(r)}$ \\
\hline$r=0$ & $\mathbf{x}^{(0)}=(0,150,50,200)$ & 1 & 0 & 0 & 0 \\
$r=1$ & $\mathbf{x}^{(1)}=(24.165,125.835,25.835,224.165)$ & 0.527 & 0.419 & 0.527 & 0.419 \\
$r=2$ & $\mathbf{x}^{(2)}=(26.51,123.49,23.49,226.51)$ & 0.48 & 0.465 & 0.574 & 0.465 \\
$r=3$ & $\mathbf{x}^{(3)}=(26.82,123.18,23.18,226.82)$ & 0.473 & 0.471 & 0.58 & 0.471 \\
$r=4$ & $\mathbf{x}^{(4)}=(26.86,123.14,23.14,226.86)$ & 0.473 & 0.472 & 0.58 & 0.472 \\
$r=5$ & $\mathbf{x}^{(5)}=(26.867,123.133,23.133,226.867)$ & 0.472 & 0.472 & 0.581 & 0.472 \\
\hline
\end{tabular}

TABLE 3: The results corresponding to two iterations for the problem (25).

\begin{tabular}{llcccc}
\hline Iteration $r$ & $x^{(r)}$ & $\mu_{1}\left(x^{(r)}\right)$ & $\mu_{2}\left(x^{(r)}\right)$ & $\mu_{3}\left(x^{(r)}\right)$ & $\lambda^{(r)}$ \\
\hline$r=0$ & $\mathbf{x}^{(0)}=(0,150,50,200)$ & 1 & 0 & 0 & 0 \\
$r=1$ & $\mathbf{x}^{(1)}=(24,126,26,224)$ & 0.530 & 0.416 & 0.524 & 0.416 \\
$r=2$ & $\mathbf{x}^{(2)}=(27,123,23,227)$ & 0.470 & 0.475 & 0.583 & 0.470 \\
\hline
\end{tabular}

that is,

$$
\begin{gathered}
\max t \\
0.001\left(-20.785 x_{11}-81.585 x_{12}+113.832 x_{21}\right. \\
\left.+35.355 x_{22}-3.107\right) \geq t \\
0.002\left(-2.982 x_{11}-5.965 x_{12}-4.151 x_{21}\right. \\
\left.+4.212 x_{22}-14.328\right) \geq t \\
0.001\left(52.753 x_{11}-14.439 x_{12}+46.785 x_{21}\right. \\
\left.-2.502 x_{22}-10.972\right) \geq t \\
x_{11}+x_{12} \leq 150, \quad x_{21}+x_{22} \leq 250 \\
x_{11}+x_{21} \geq 50, \quad x_{12}+x_{22} \geq 350 \\
\mathbf{x}=\left(x_{11}, x_{12}, x_{21}, x_{22}\right) \geq \mathbf{0}
\end{gathered}
$$

Solving this problem, we obtain $\mathbf{x}^{(2)}=$ $\left(x_{11}, x_{12}, x_{21}, x_{22}\right)=(26.510,123.490,23.490,226.510)$.

The results corresponding to five iterations for the problem (25) are given in Table 2. Table 2 shows number of iterations $r$, the satisfactory levels of the objectives corresponding to solution $\mathbf{x}^{(r)}$, and the monotone increasing sequence $\lambda^{(r)}$.

For $r=5$ and $t=0.0004<\delta=10^{-3}$ in Step 2, then the current solution $\mathbf{x}^{*}=\mathbf{x}^{(r)}=x^{(5)}=(26.867,123.133,23.133$, 226.867 ) is a compromise solution of the problem (25) ("min" operator model), and the values of membership functions are $\mu_{1}\left(\mathbf{x}^{(5)}\right)=0.472, \mu_{2}\left(\mathbf{x}^{(5)}\right)=0.472$, and $\mu_{3}\left(\mathbf{x}^{(5)}\right)=0.581$ respectively. The optimal value of max-min problem is $\lambda^{(r)}=$ $\lambda^{(5)}=0.472$ and can also be interpreted as the most basic satisfactory level of each objective in this transportation system can attain. Due to the Pareto-optimality test, this compromise solution is also a Pareto-optimal solution because there does not exist alternative solution of problem (25).

Remark 10. As known, unimodularity feature is observed in the coefficient matrix of the constraints of transportation problem where the determinant of all the square submatrices is either 0 or +1 or -1 . If this feature is not satisfied, then the integer solution does not guarantee and the problem does not fit the standard form of the transportation problem [36].

In our fuzzy approach, model (13) or max-min model is a linear fractional compromise model and it does not fit the standard form of the transportation problem because of membership constraints $\mu_{q}\left(z_{q}\right) \geq \lambda$ or multiple-objective functions $z_{q}, q=1, \ldots, Q$. The coefficient matrix of the constraints of MLFTP in a canonical form will not be unimodular, and consequently integer solution does not guarantee. Table 2 shows these results obtained by ignoring unimodularity property or the integrality restrictions. If we want to obtain a strictly integer solution for the given fuzzy approach, integer programming is used to overcome the nonintegrity problem. That is, we must put integer conditions on decision variables in solution algorithm. In this case, to reach an integer compromise Pareto-optimal solution for the problem (25), two iterations are required and the results corresponding to them are given in Table 3.

Remark 11. All solutions are obtained by using the GAMS computer package.

\section{Conclusion}

In this study, MLFTP has been introduced for the first time according to the best of our knowledge. First, we provided a theorem emphasizing the fact that MLFTP always has feasible solution; its set of feasible solutions is bounded, and, hence, MLFTP is always solvable. It is also proposed a fuzzy approach which generates a compromise Paretooptimal solution for MLFTP by reducing the problem to the Zimmermann's "min" operator model and then constructing a solution algorithm based on Generalized Dinkelbach's Algorithm.

\subsection{Advantages of Our Method}

(i) By means of Generalized Dinkelbach's Algorithm, MLFTP with nonlinear structure is reduced to a 
sequence of linear problems. Using LP is one of the most advantageous aspects of this method. We note that, although choosing linear membership function gives essential cause for this linearization property, using another different shape of membership functions such as hyperbolic and exponential and piecewise-linear, by means of Generalized Dinkelbach's Algorithm, does not.

(ii) Our method generates a weakly Pareto-optimal solution for MLFTP, if the problem has alternative solutions. In this case, by using the Pareto-optimality test, a compromise Pareto-optimal solution is found. Otherwise, our method can directly find a compromise Pareto-optimal solution.

(iii) The sequence $\left\{\lambda^{r}\right\}$ for all $r \geq 0$ generated by our algorithm is nondecreasing and bounded above by $\lambda^{*}$ $\left(\lambda^{*}=\max _{\mathbf{x} \in S} \min _{1 \leq q \leq Q} \mu_{q}\left(z_{q}(\mathbf{x})\right)\right)$, since MLFTP is solvable.

An illustrative example is presented to show our fuzzy approach. As seen, at each iteration LP problems were solved rather than linear fractional ones.

5.2. Future Directions. This paper mainly provided an introduction to MLFTP. Many areas are still needed to be explored and developed in this direction. Some fuzzy approachesusing the goal programming, and/or using the nonlinear membership functions - can be developed for MLFTP, to find its compromise Pareto-optimal solution. Computer codes must be written to be used to solve large-scale applications of the method, such as applications to transportation system problems. Moreover, fuzzy approaches with fuzzy parameters for MLFTP together with different shapes of membership functions and/or their stochastic models, solid transportation problems, network problems, and so forth may become new topics in further research.

\section{Conflict of Interests}

The authors declare that there is no conflict of interests regarding the publication of this paper.

\section{Acknowledgments}

The authors are very indebted to the anonymous referees for their critical suggestions for improvements.

\section{References}

[1] L. A. Zadeh, "Toward a generalized theory of uncertainty (GTU)- an outline," Information Sciences, vol. 172, no. 1-2, pp. $1-40,2005$.

[2] L. A. Zadeh, "Is there a need for fuzzy logic?" Information Sciences, vol. 178, no. 13, pp. 2751-2779, 2008.

[3] B. B. Pal and S. Gupta, "A genetic algorithm approach to fuzzy goal programming formulation of fractional multiobjective decision making problems," in Proceedings of the 1st International Conference on Advanced Computing (ICAC '09), pp. 5560, December 2009.
[4] A. Charnes and W. W. Cooper, "Programming with linear fractional functionals," Naval Research. Logistics Quarterly, vol. 9, pp. 181-186, 1962.

[5] G. R. Bitran and A. G. Novaes, "Linear programming with a fractional objective function," Operations Research, vol. 21, pp. 22-29, 1973.

[6] J. S. H. Kornbluth and R. E. Steuer, "Goal programming with linear fractional criteria," European Journal of Operational Research, vol. 8, no. 1, pp. 58-65, 1981.

[7] J. S. H. Kornbluth and R. E. Steuer, "Multiple objective linear fractional programming," Management Science, vol. 27, no. 9, pp. 1024-1039, 1981.

[8] I. Nykowski and Z. Zołkiewski, "A compromise procedure for the multiple objective linear fractional programming problem," European Journal of Operational Research, vol. 19, no. 1, pp. 9197, 1985.

[9] F. Tiryaki, Solution proposals to multi-objective linear fractional programming problem [Ph.D. thesis], Yildiz Technical University, Institution of Science, 1993.

[10] M. K. Luhandjula, "Fuzzy approaches for multiple objective linear fractional optimization," Fuzzy Sets and Systems, vol. 13, no. 1, pp. 11-23, 1984.

[11] M. Sakawa and T. Yumine, "Interactive fuzzy decision-making for multiobjective linear fractional programming problems," Large Scale Systems, vol. 5, pp. 105-113, 1983.

[12] M. Sakawa and H. Yano, "An interactive fuzzy satisficing method for multiobjective linear fractional programming problems," Fuzzy Sets and Systems, vol. 28, no. 2, pp. 129-144, 1988.

[13] M. Chakraborty and S. Gupta, "Fuzzy mathematical programming for multi objective linear fractional programming problem," Fuzzy Sets and Systems, vol. 125, no. 3, pp. 335-342, 2002.

[14] A. K. Bit, M. P. Biswal, and S. S. Alam, "Fuzzy programming approach to multicriteria decision making transportation problem," Fuzzy Sets and Systems, vol. 50, no. 2, pp. 135-141, 1992.

[15] A. K. Bit, M. P. Biswal, and S. S. Alam, "An additive fuzzy programming model for multiobjective transportation problem," Fuzzy Sets and Systems, vol. 57, no. 3, pp. 313-319, 1993.

[16] R. Verma, M. P. Biswal, and A. Biswas, "Fuzzy programming technique to solve multi-objective transportation problems with some non-linear membership functions," Fuzzy Sets and Systems, vol. 91, no. 1, pp. 37-43, 1997.

[17] S. K. Das, A. Goswami, and S. S. Alam, "Multiobjective transportation problem with interval cost, source and destination parameters," European Journal of Operational Research, vol. 117, no. 1, pp. 100-112, 1999.

[18] W. F. A. El-Wahed, "A multi-objective transportation problem under fuzziness," Fuzzy Sets and Systems, vol. 117, no. 1, pp. 2733, 2001.

[19] L. Li and K. K. Lai, "A fuzzy approach to the multiobjective transportation problem," Computers and Operations Research, vol. 27, no. 1, pp. 43-57, 1999.

[20] S.-T. Liu and C. Kao, "Solving fuzzy transportation problems based on extension principle," European Journal of Operational Research, vol. 153, no. 3, pp. 661-674, 2003.

[21] E. E. Ammar and E. A. Youness, "Study on multiobjective transportation problem with fuzzy numbers," Applied Mathematics and Computation, vol. 166, no. 2, pp. 241-253, 2005.

[22] W. F. Abd El-Wahed and S. M. Lee, "Interactive fuzzy goal programming for multi-objective transportation problems," Omega, vol. 34, no. 2, pp. 158-166, 2006. 
[23] M. Jain and P. K. Saksena, "Time minimizing transportation problem with fractional bottleneck objective function," Yugoslav Journal of Operations Research, vol. 22, pp. 115-129, 2012.

[24] E. B. Bajalinov, Linear Fractional Programming: Theory, Methods, Applications and Software, Kluwer Academic, Boston, Mass, USA, 2003.

[25] Z. Zenzerovic, D. Sc, and S. Beslic, "Optimization of cargo transport with a view to cost efficient operation of container ship," in Proceedings of the 25th International Conference Information Technology Interfaces, 2003.

[26] H. Leberling, "On finding compromise solutions in multicriteria problems using the fuzzy min-operator," Fuzzy Sets and Systems, vol. 6, no. 2, pp. 105-118, 1981.

[27] F. Tiryaki, "Interactive compensatory fuzzy programming for decentralized multi-level linear programming (DMLLP) problems," Fuzzy Sets and Systems, vol. 157, no. 23, pp. 3072-3090, 2006.

[28] M. Sakawa, Fuzzy Sets and Interactive Multiobjective Optimization, Plenum Press, New York, NY, USA, 1993.

[29] H.-J. Zimmermann, "Fuzzy programming and linear programming with several objective functions," Fuzzy Sets and Systems, vol. 1, no. 1, pp. 45-55, 1978.

[30] J. Borde and J. P. Crouzeix, "Convergence of a Dinkelbach-type algorithm in generalized fractional programming," Zeitschrift für Operations Research, vol. 31, no. 1, pp. A31-A54, 1987.

[31] H. Katagiri, M. Sakawa, and H. Ishii, "Multiobjective fuzzy random linear programming using E-model and possibility measure," in Proceedings of the Joint 9th IFSA World Congress and 20th NAFIPS International Conference, pp. 2295-2300, July 2001.

[32] B. Elbenani and J. A. Ferland, Cell Formation Problem Solved Exactly with the Dinkelbach Algorithm, CIRRELT, 2012.

[33] J. P. Crouzeix, J. A. Ferland, and S. Schaible, "An algorithm for generalized fractional programs," Journal of Optimization Theory and Applications, vol. 47, no. 1, pp. 35-49, 1985.

[34] M. Ahlatcioglu and F. Tiryaki, "Interactive fuzzy programming for decentralized two-level linear fractional programming (DTLLFP) problems," Omega, vol. 35, no. 4, pp. 432-450, 2007.

[35] R. E. Rosenthal, GAMS-A User's Guide, GAMS Development Corporation, Washington, DC, USA, 2007.

[36] R. K. Ahuja, T. L. Magnanti, and J. B. Orlin, Network Flows: Theory, Algorithms, and Applications, Prentice Hall, Englewood Cliffs, NJ, USA, 1993. 


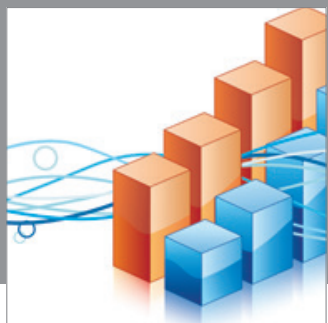

Advances in

Operations Research

mansans

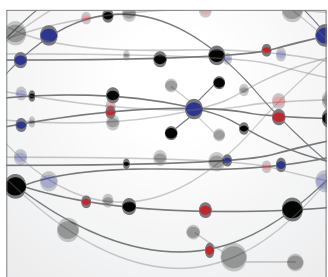

The Scientific World Journal
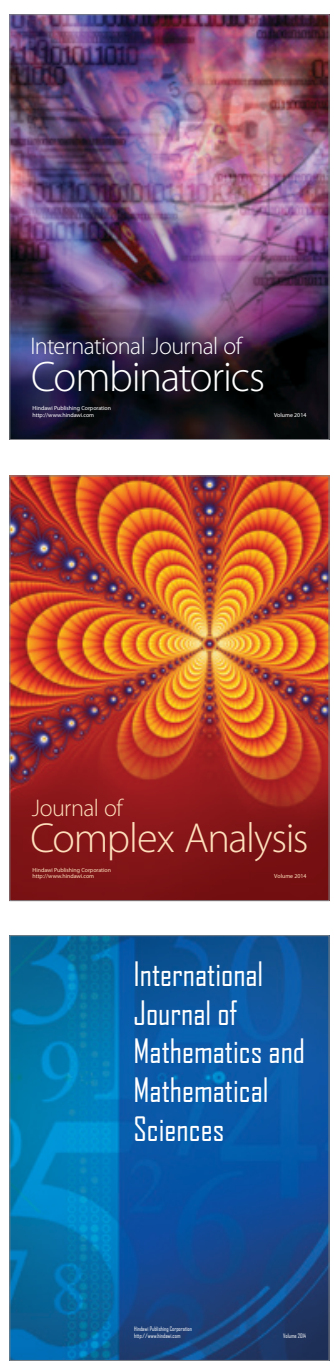
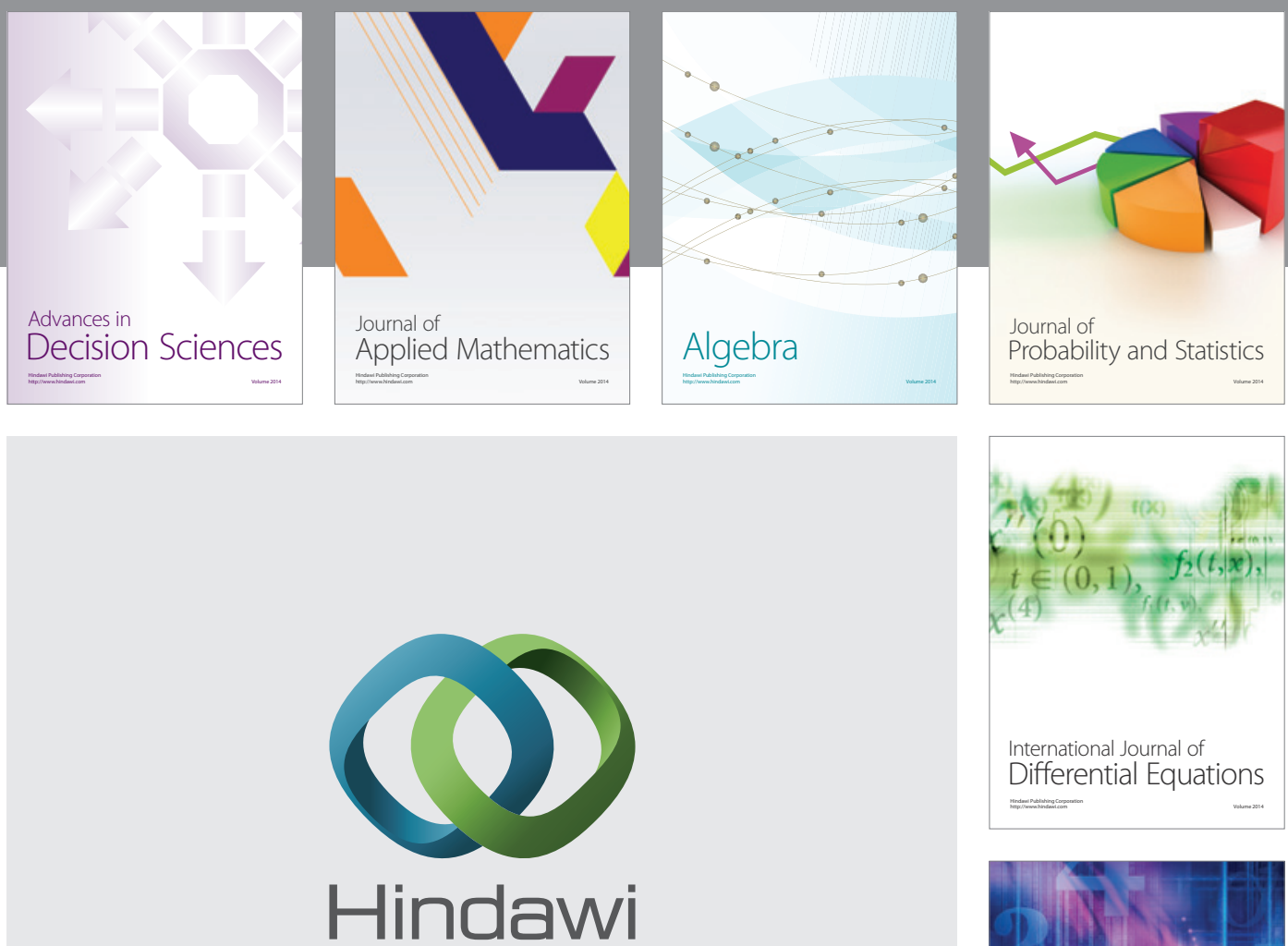

Submit your manuscripts at http://www.hindawi.com
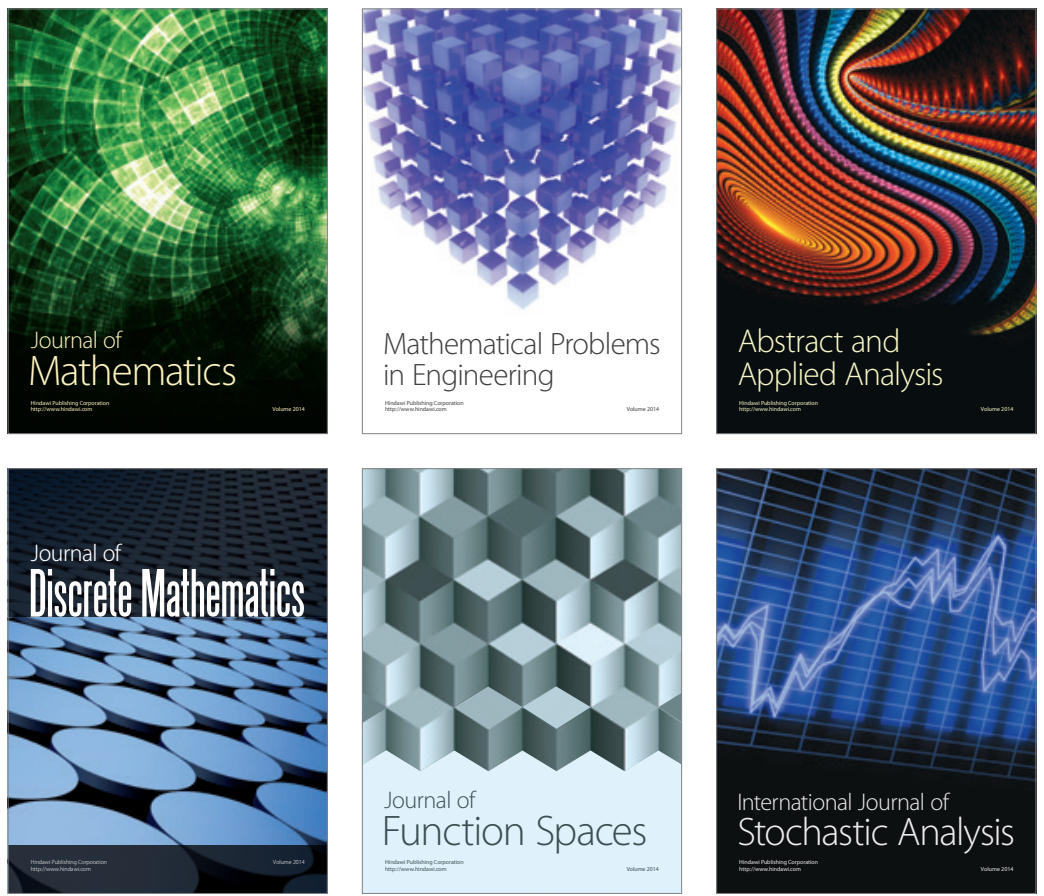

Journal of

Function Spaces

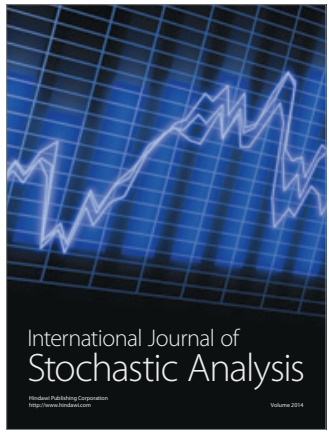


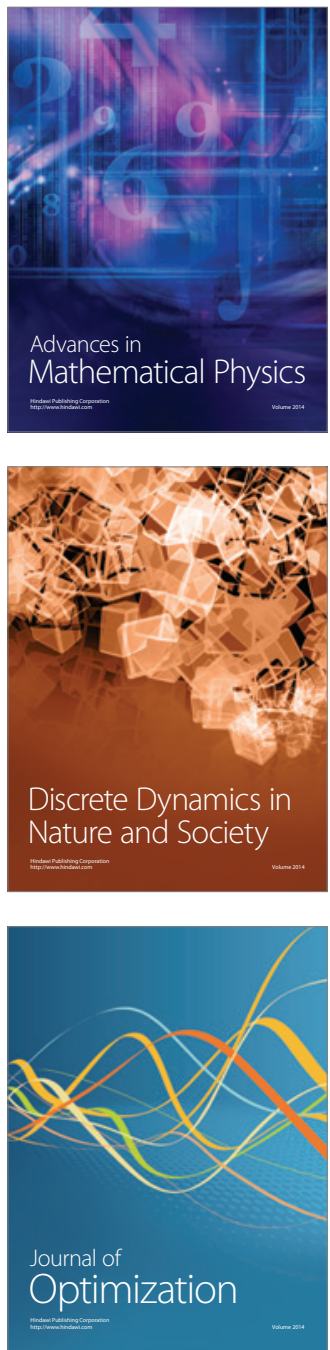